\title{
Dapagliflozin: A Novel Therapeutic Approach to Treat Diabetes
}

\author{
Suruchi Aditya, M.D., 'SK Mathur, M.D., ${ }^{2}$ Aditya Rattan, M.D. ${ }^{3}$ \\ 'Department of Pharmacology, Dr HSJIDS, Sec 25,Chandigarh, ${ }^{2}$ Department of Pediatrics, M.M Institute of Medical Sciences, Mullana, \\ Ambala, ${ }^{3}$ Consultant Cardiologist, Heartline, SCO-58, Sector 6, Panchkula
}

\section{A B S T R A C T}

Current approaches to treatment of diabetes have many drawbacks. Most of the oral hypoglycemic drugs target the insulin secretion and action. Sodium Glucose co transporters (SGLT)-2 inhibitors are a recent addition to antihyperglycemic drugs that act by reducing glucose reabsorption from the renal filtrate so that bulk of glucose is excreted in the urine. Dapagliflozin, the first in this new class of drugs, is a SGLT2 inhibitor. It causes loss in body weight and is not associated with major hypoglycemic events. Dapagliflozin is currently in advanced development for use alone or in combination with other hypoglycemic agents. Concerns regarding increased cancer risk have delayed the Food and Drug Authority (FDA) approval of this drug. SGLT2 inhibitors represent a major advancement in management of type 2 diabetes mellitus (T2DM) as they have shifted the focus onto kidney and act independent of insulin. JMS $2011 ; 14(2): 43-45$

Key Words: SGLT2 inhibitors, dapagliflozin, type 2 diabetes

Diabetes is a healthcare crisis affecting 51 million Indians- the highest number of diabetics in any one country in the entire world. ${ }^{1}$ Worldwide, the diabetic population is projected to reach 440 million by 2030. Current treatment options for diabetes target the insulin pathway and are not only unsatisfactory but also have unacceptable adverse effects. Only $7-15 \%$ of patients are able to meet their glycemic target. Moreover, most of the agents lose effectiveness as pancreatic $\beta$ cell function declines.

By playing a role in gluconeogenesis and by regulating glucose excretion, kidney occupies a central role in glucose homeostasis. SGLTs are a family of membrane proteins that accomplish the task of transport of glucose from the tubules to

\footnotetext{
Correspondence and Reprint Requests to:

Dr. Suruchi Aditya

Sr. Lecturer, Department of Pharmacology,

Dr. Harvansh Singh Judge Institute of Dental Sciences,

Panjab University, Sector 25, Chandigarh.

Phone number - 9530669929

E-mail address - suruchiaditya@rediffmail.com
}

peritubular capillaries via tubular epithelial cells. SGLT1 is the key transporter for glucose absorption in the gastrointestinal tract (GIT) and accounts for $10 \%$ of glucose reabsorption in kidney. SGLT2, located primarily in S1 segment of proximal tubule, is responsible for $90 \%$ of glucose reabsorption by kidney, using the energy gradient of sodium reabsorption in the tubular filtrate. Diabetes patients show increased expression and activity of SGLT2 leading to increased tubular reabsorption of glucose. $^{2}$

Inhibiting SGLT2 is a novel therapeutic approach to treat diabetes. It not only corrects a defective mechanism in diabetics but also promotes weight loss by causing glycosuria (1g glucose is equivalent to $4 \mathrm{Kcal}$ ). Non selective SGLT inhibitors cause osmotic diarrhea limiting their clinical utility. Novel SGLT2 inhibitors are Dapagliflozin, Canagliflozin, Sergliflozin and Remogliflozin etabonate.

The parent compound of this new class of drugs is phlorizin- an O-glucoside. The O-glucosides have to be administered as their prodrug esters to avoid degradation by $\beta$ glucosidase in the small intestine. Novel SGLT2 inhibitors are 
C- glucosides; creating a carbon- carbon bond between the glucose and aglycone moiety converts $\mathrm{O}$ - glucosides to Cglycosides, making them unsusceptible to $\beta$ glucosidase. ${ }^{3}$

\section{Mechanism of action}

Dapagliflozin (Fig. 1) is 1200 times more selective for SGLT2 over SGLT1. SGLT2 inhibition involves competitive binding of glucose moiety binding site on the transport protein and results in reduction of plasma glucose levels by reducing the glucose Tm (transport maximum) due to increased urinary excretion. Though not a class effect, SGLT2 inhibition also causes reduction in hepatic gluconeogenesis and decreased glucotoxicity. By lowering plasma glucose, liver sensitivity is improved which leads to suppression of hepatic glucose production as a result of glucose 6 phosphatase inhibition. ${ }^{4}$

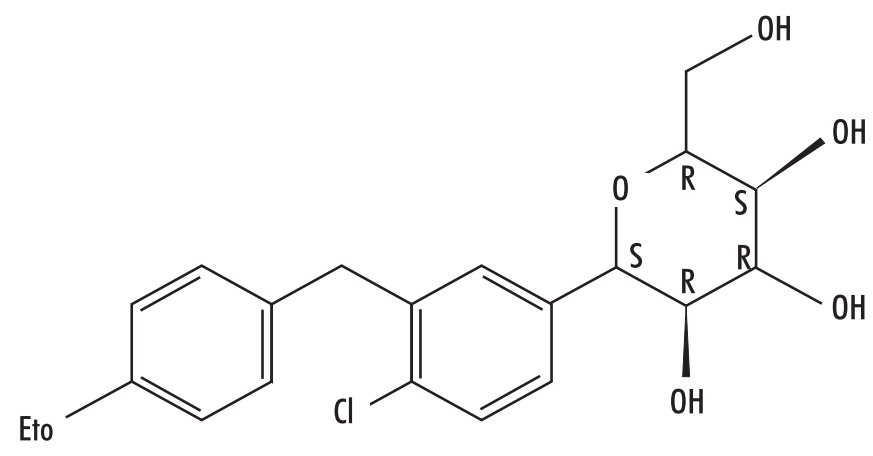

FIGURE 1: Molecular structure of dapagliflozin

By causing sodium excretion and possibly by a diuretic effect, these drugs can cause a mild reduction in arterial blood pressure. ${ }^{4}$

\section{Clinical studies done with dapagliflozin}

In a 24 week Phase III study done on more than 1200 patients, by Astrazeneca and Bristol Myers Squibb ${ }^{5}, 2$ distinct groups were studied using dapagliflozin $5 \mathrm{mg}$ and $10 \mathrm{mg}$. Each group comprised three categories-metformin plus dapagliflozin, dapagliflozin plus placebo and metformin plus placebo.

In both these groups, combination of metformin and dapagliflozin produced maximum reduction in HbA1C [-2.05\% versus (vs)-1.98\% with dapagliflozin $5 \mathrm{mg}$ and $10 \mathrm{mg}$ respectively] than either of the drugs combined with a placebo. The combination group showed significantly greater decrease in fasting plasma glucose $(-61.0 \mathrm{mg} / \mathrm{dL}$ and $-60.4 \mathrm{mg} / \mathrm{dL}$ with dapagliflozin 5mg and $10 \mathrm{mg}$ respectively). Similarly, mean reduction in body weight was maximum in metformin plus dapagliflozin category (-2.66 kg vs-3.33 kg with dapagliflozin $5 \mathrm{mg}$ and $10 \mathrm{mg}$ respectively) in both these groups. Incidence of genital infections $(12.8 \%$ and $6.9 \%$ in dapagliflozin 10 and $5 \mathrm{mg}$ respectively) and urinary tract infections (UTIs) $(11 \%$ and $7.9 \%$ in dapagliflozin 10 and $5 \mathrm{mg}$ respectively) were highest in dapagliflozin plus placebo category in either group.

An extension of the study evaluated the drug upto102 weeks and found that the primary endpoint - change from baseline in $\mathrm{HbA1C}$ in patients receiving placebo plus metformin was $-0.02 \%$ compared to $-0.58 \%$ for patients receiving dapagliflozin $5 \mathrm{mg}$ plus metformin and $-0.78 \%$ for patients receiving dapagliflozin $10 \mathrm{mg}$ plus metformin. ${ }^{6}$ These studies concluded that dapagliflozin is more efficacious than metformin alone.

A 2 year data from a randomized controlled trial (RCT) evaluated dapagliflozin (up to $10 \mathrm{mg} / \mathrm{d}, \mathrm{n}=406$ ) vs. glipizide (upto $20 \mathrm{mg} / \mathrm{d}, \mathrm{n}=408$ ) as add on therapy to metformin (up to $2 \mathrm{~g} /$ day). ${ }^{7}$ A decline in HbA1C levels of $0.32 \%$ [ $95 \%$ confidence interval (CI), $-0.42 \%$ to- $0.21 \%$ ] with dapagliflozin vs. $0.14 \%(95 \%$ CI, $-0.25 \%$ to $-0.03 \%)$ with glipizide was observed. Dapagliflozin also produced a sustained reduction in body weight $(-3.70 \mathrm{~kg}$ vs. $+1.36 \mathrm{~kg})$ and low risk of hypoglycemia (dapagliflozin $4.2 \%$ vs. glipizide $45.8 \%$ ) over a course of 2 years.

Another phase III double blind placebo controlled trial of dapagliflozin as an add on agent examined 546 poorly controlled T2DM patients on maximum doses of metformin, randomized to receive dapagliflozin $2.5 \mathrm{mg} 5 \mathrm{mg}, 10 \mathrm{mg} /$ day or placebo. At the end of 24 weeks, patients on dapagliflozin $5 \mathrm{mg}$ showed $-0.70 \%$ change in HbA1C from baseline vs. $-0.84 \%$ with dapagliflozin $10 \mathrm{mg}$ and $-0.3 \%$ with placebo $(p<0.002$ for both comparisons). Moreover, dapagliflozin 5 and $10 \mathrm{mg}$ showed significant fall in systolic and diastolic blood pressure. ${ }^{8}$

A small study examined effect of dapagliflozin in patients of T2DM who were experiencing suboptimal control on insulin and insulin sensitizers (pioglitazone $>30 \mathrm{mg}$ / rosiglitazone $4 \mathrm{mg} / \mathrm{metformin}>1000 \mathrm{mg})$. Change in HbA1C from baseline at week 12 was reported to decrease $0.7 \%$ with 10 mg dapagliflozin and $0.78 \%$ with $20 \mathrm{mg}$ dapagliflozin.

\section{Adverse effects}

Side effects observed in clinical studies done on dapagliflozin apart from diarrhea and nausea are urinary tract and genital tract infections, dizziness, headache, fatigue, backache and nasopharyngitis. ${ }^{10}$ Increased urine volume was associated with increased hematocrit and urea, suggesting slight volume depletion. Electrolyte imbalance is a consideration because physiological studies show increased sodium loss with phlorizin. ${ }^{3}$ Statistically significant increases in serum magnesium and decreased uric acid levels have been reported. An increase in parathormone concentration but no changes in 1,25 dihydroxy vitamin D and 25- hydroxy vitamin D levels have been noted. ${ }^{13}$ Dapagliflozin was associated with a higher rate of genital infections as compared to metformin plus placebo group (12.8\% vs. $2.4 \%$ ) and UTIs (11\% vs. $4.3 \%){ }^{6}$

There have been no reports of major hypoglycemic episodes and no patient discontinued study medication due to hypoglycemia. Drug induced liver injury is a concern because some C-glycoside SGLT2 inhibitors reported a positive 
micronucleus test-indicating a potential to damage chromosomes. This observation has encouraged development of new phlorizin analogues by slight modification of chemical structure. Further evaluations are needed to clarify its effects in diabetes patients with neuropathy, hyporeninemic hypoaldosteronism, nephropathy and those vulnerable to sodium wasting and hypovolemia. Safety in pregnancy is not established.

In clinical trials, out of 5478 patients, 9 patients $(0.1 \%)$ had breast cancer, 9 patients $(0.35 \%)$ had bladder cancer compared with only one breast cancer $(0.09 \%)$ and 1 bladder cancer $(0.5 \%)$ among 3156 patients assigned to control groups. As reporting of cancer was within one year of drug initiation in all patients, it seems unlikely that dapagliflozin caused the cancer, although it might have accelerated them. Moreover, dapagliflozin has not shown genotoxic or carcinogenic potential in preclinical studies. Due to concerns over these serious adverse effects, FDA recently declined approval of this drug asking the developers to provide more data about its riskbenefit profile.

\section{Conclusion}

The clinical trials done on dapagliflozin either alone or in combination with various antidiabetic drugs have shown promising results in reducing $\mathrm{HbA} 1 \mathrm{C}$; this drug in future could prove to be an additional molecule in diabetes management. The studies are on to evaluate the adverse effects of the drug. Phase III trials till date have not reported any serious adverse events precluding the clinical use of this molecule. Further ongoing studies will enrich our knowledge about this drug. More RCTs are required to study the long term impact in diabetes management. Though yet to obtain FDA approval; this new class of drugs is poised to play an important role in the treatment of diabetes as they work independently of insulin and target renal glucose reabsorption.

\section{References}

1. Anjana RM, Ali MK, Pradeepa R, Deepa M, Datta M, Unnikrishnan $\mathrm{R}$, et al. The need for obtaining accurate nationwide estimates of diabetes prevalence in IndiaRationale for a national study on diabetes prevalence in India. Indian J Med Res 2011;133:369-380.

2. Perez Lopez G, Gonzalez Albarran O, Cano Megias M.
Type 2 sodium glucose cotransporter (SGLT2) inhibitors: from familial renal glucosuria to the treatment of type 2 diabetes mellitus. Nefrologia 2010; 30 (6):618-25.

3. Nair S and WildingJPH. Sodium glucose contrasporter 2 inhibitors as a new treatment for diabetes mellitus. J Clin Endocrinol Metab 2010;95(1):34-42.

4. Boldys A, Okopien B. Inhibitors of type 2 sodium glucose co-transporter: a new strategy for diabetes treatment. Pharm Rep 2009;61:778-84.

5. Astrazeneca, Bristol - Myers Squibb. Investigational compound dapagliflozin with metformin extended release (XR) as initial combination therapy significantly improved blood sugar control in previously- untreated adult type 2 diabetes patients with high blood sugar levels. Available from www. Astrazeneca-us.com/ aboutastrazeneca-us/12379184.

6. Bristol Myers Squibb and Astrazeneca. Bristol Myers Squibb and AstraZeneca announce investigational compound dapagliflozin sustained glycemic control and weight reduction in study of Type 2 diabetes patients inadequately controlled with metformin. Available from :http://www.astrazeneca.com/ Research / news/ Article/ 27062011

7. Nauck MA, Del Prato S, Meier JJ, Duran-Garcia S, Rohwedder K, Elze M, et al. Dapagliflozin versus glipizide as add-on therapy in patients with type 2 diabetes who have inadequate glycemic control with metformin: a randomized, 52-week, double-blind, active-controlled noninferiority trial. Diabetes Care 2011; 34:2015-2022.

8. Bailey CJ, Gross JL, Pieters A, Bastier A, List JF. Effect of dapagliflozin in patients with type 2 diabetes who have inadequate glycemic control with metformin: a randomised, double- blind, placebo- controlled trial. Lancet 2010; 3756 (9733): 2223-33.

9. Wilding JP, Norwood P, T Joen C, Bastien A, List JF, Fiedorek FT. A study of dapagliflozin in patients with type 2 diabetes receiving high doses of insulin plus insulin sensitizers: applicability of novel insulin - independent treatment. Diabetes Care 2009;32(9):1656-62.

10. List JF, Woo V, Morales E, Tang W, Fieldorek FT. Sodium glucose co-transport inhibition with dapagliflozin in type 2 diabetes mellitus. Diabetes Care 2009;32:650-7. 